\title{
Intraoperative Ultrasound in Patients with Hepatocellular Carcinoma: From Daily Practice to Future Trends
}

\author{
Matteo Donadon Guido Torzilli \\ Liver Surgery Unit, Department of General Surgery, University of Milan School of Medicine, \\ Humanitas Cancer Center, Rozzano, Italy
}

\section{Key Words}

Intraoperative ultrasound $\cdot$ Hepatocellular carcinoma $\cdot$ Tumor limits

\section{Abstract}

Starting from the end of the 1970s, intraoperative ultrasound was introduced and developed to stage liver disease and guide the surgical interventions in patients with hepatocellular carcinoma. Without intraoperative ultrasound, it would probably be impossible to correctly define the hepatic segments as well as the limits of a tumor, also because of the existing wide variations in the anatomy. More importantly, intraoperative ultrasound allows for three-dimensional reconstruction of the relationships between the tumor, hepatic veins, and Glissonian pedicles. Such reconstruction is essential for planning the surgical strategy, which should be individualized in each patient with the aim to spare functional liver parenchyma. This work focuses on the role of intraoperative ultrasound to stage liver disease and to plan surgical resection, describing the most recent technical achievements.

Copyright $(2013$ S. Karger AG, Basel

\section{Introduction}

Since the end of the 1970s, intraoperative ultrasound (IOUS) has been used to stage liver disease and to guide the surgical interventions in patients with hepatocellular carcinoma (HCC) and underlying cirrhosis [1-4]. The burgeoning use of IOUS started in Japan and from 


\section{Liver Cancer}

\begin{tabular}{|c|c|}
\hline \multicolumn{2}{|l|}{ Liver Cancer 2013;2:16-24 } \\
\hline $\begin{array}{l}\text { DOI: } 10.1159 / 000346421 \\
\text { Published online: January 7, } 2013\end{array}$ & $\begin{array}{l}\text { (c) } 2013 \text { S. Karger AG, Basel } \\
\text { www.karger.com/lic }\end{array}$ \\
\hline
\end{tabular}

there it spread worldwide allowing for the development of different therapies to treat patients with HCC [5]. This work focuses on the role of IOUS to stage liver disease and to plan surgical resection, describing the most recent technical achievements.

\section{Who Should Perform IOUS?}

It is worth mentioning that IOUS should be performed by the surgeon in charge of the operation rather than by assistants, radiologists, or technicians as the information gathered during the exploration requires interpretation to be most profitable with the aim to impact the surgical strategy. Indeed, nowadays IOUS is equally performed to plan the surgical strategy and to disclose the lesions. Therefore, the first surgeon should master ultrasound anatomy and techniques with the aim to modify the operative strategy in real time whenever required. In particular, patients with chronic hepatitis or cirrhosis demand a parenchymasparing liver surgery to minimize the risk of postoperative liver failure, which still represents an important source of mortality $[6,7]$.

\section{Technical Equipment}

Generally, high-frequency probes $(7.5-10 \mathrm{MHz})$ are recommended to perform IOUS since they allow for a higher spatial resolution than those working at lower frequencies (3.5-5 MHz). However, those latter probes are very useful for the initial exploration providing a better panoramic view. Lower-frequency probes are also useful for allowing contrastenhanced intraoperative ultrasonography. The optimal probe in this sense should be the one working within a wide range of frequencies. Different shapes of probes are available for intraoperative use: linear T-shaped probes, interdigital probes, microconvex probes, and, more recently, T-shaped probes with trapezoid scanning window. The best probe is the one that ensures the optimal compromise between the volume of the probe itself, which should be minimal, the scanning windows, which should be the largest, and the stability once in contact with the liver surface. In this sense, microconvex probes and linear probes with trapezoid scanning represent the optimal solutions (fig. 1). Of note, the performance of IOUS may take time and requires experience to be effective and profitable [8].

\section{Examination Technique}

The ultrasound machine (fig. 2) should be placed in front of the first operator, with the aim to simultaneously view the screen and the operative field. The screen must be large enough to allow optimal visibility at that distance, and the assistants should then control the machine's keyboard that can be covered with a sterile drape. The liver exploration should always start with the inspection and palpation of the organ together with the entire peritoneal cavity. This should not be avoided in favor of IOUS because, as recently demonstrated by Hata at al. [9], the inspection and palpation of the liver still play a significant role. Once in the peritoneal cavity, liver mobilization starts with the division of the round and falciform ligaments as well as of adhesions to free the anterior-superior and inferior surfaces of the liver in order to get enough space to handle the probes for IOUS. Thus, by pulling the round ligament, the liver surface is widely exposed, and following the examination of the portal branches and hepatic veins, the entire liver can be studied. 
Donadon et al.: Intraoperative Ultrasound in Patients with Hepatocellular Carcinoma: From Daily Practice to Future Trends
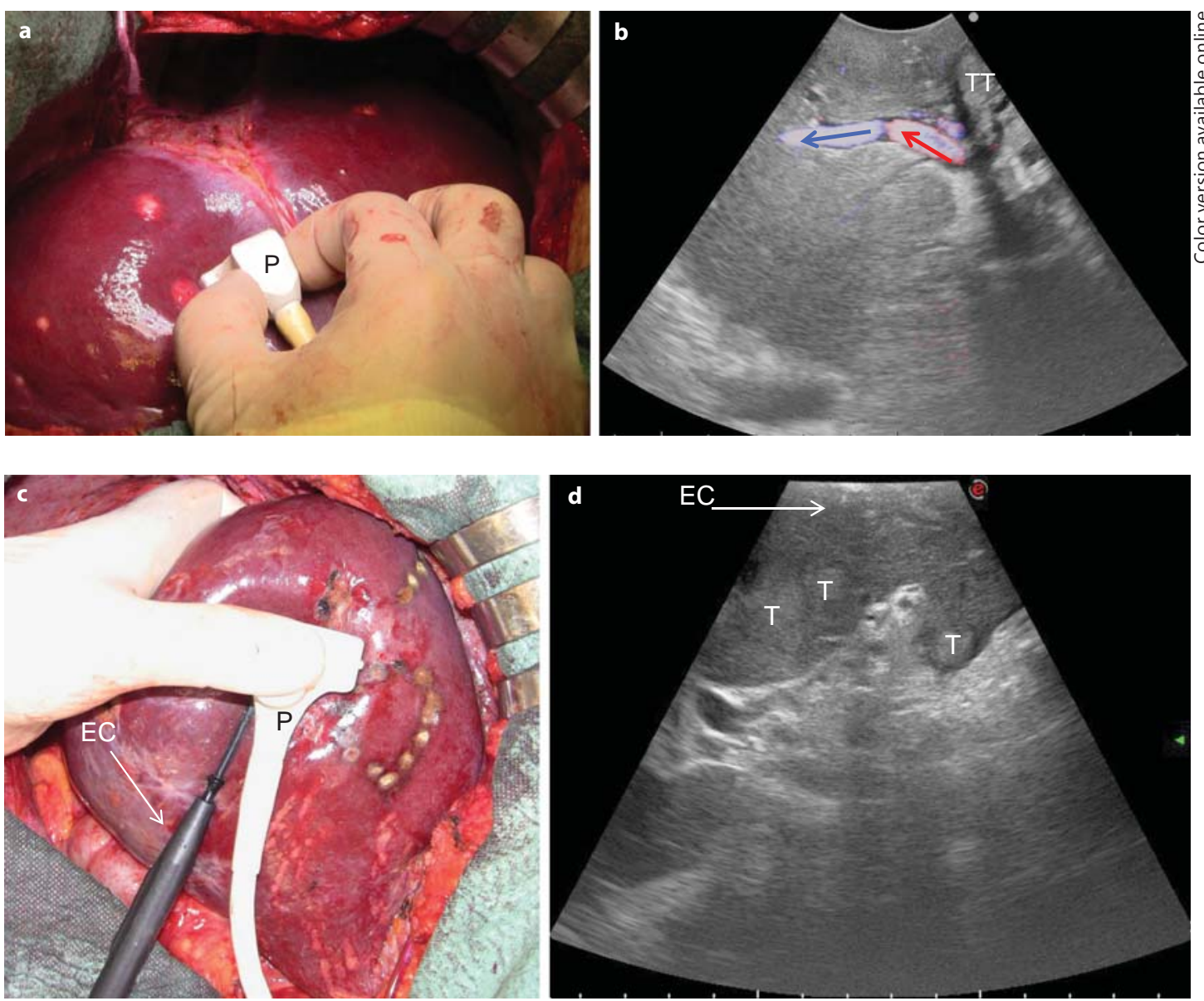

Fig. 1. a, b The microconvex probe (P) handled by the surgeon on the liver surface (a) and the corresponding ultrasound image with a wide scanning window and showing the B-mode image together with a color flow analysis (b; colored arrows indicate the blood flow direction). c, d A new T-shaped probe designed for easy handling by the surgeon to comfortably allow maneuvers: the interposition of the electrocautery (EC) for resection area demarcation (c) and an enlarged scanning window (d). $\mathrm{T}=$ Tumor; $\mathrm{TT}=$ tumor thrombus.

\section{Staging of HCC}

In the modern era of multimodality treatments for patients with HCC, the precise pretreatment staging of the disease is of paramount importance. While waiting for some more specific and individualized molecular factors, the tumor number, tumor size, their location in the liver as well as the morphology of the underlying liver, together with other important parameters such as patient age, etiology, and residual functional reserve, represent the main clinical parameters that are taken into consideration to favor one therapy over another. In particular, in patients indicated for surgery the pretreatment staging should be very accurate to avoid oncologically useless and potentially risky operations.

\section{IOUS}

Nowadays, the literature mainly supports the use of magnetic resonance imaging (MRI) over the use of computed tomography (CT) for the diagnosis and staging of HCC [10]. MRI with liver-specific contrast media allows for dynamic and functional studies of the liver 


\section{Liver Cancer}

Fig. 2. One of the most recent ultrasound machines.

\begin{tabular}{l|l}
\hline Liver Cancer 2013;2:16-24 \\
\hline DOI: 10.1159/000346421 & $\begin{array}{l}\text { ( ) 2013 S. Karger AG, Basel } \\
\text { www.karger.com/lic }\end{array}$ \\
Published online: January 7, 2013 &
\end{tabular}

Donadon et al.: Intraoperative Ultrasound in Patients with Hepatocellular Carcinoma: From Daily Practice to Future Trends

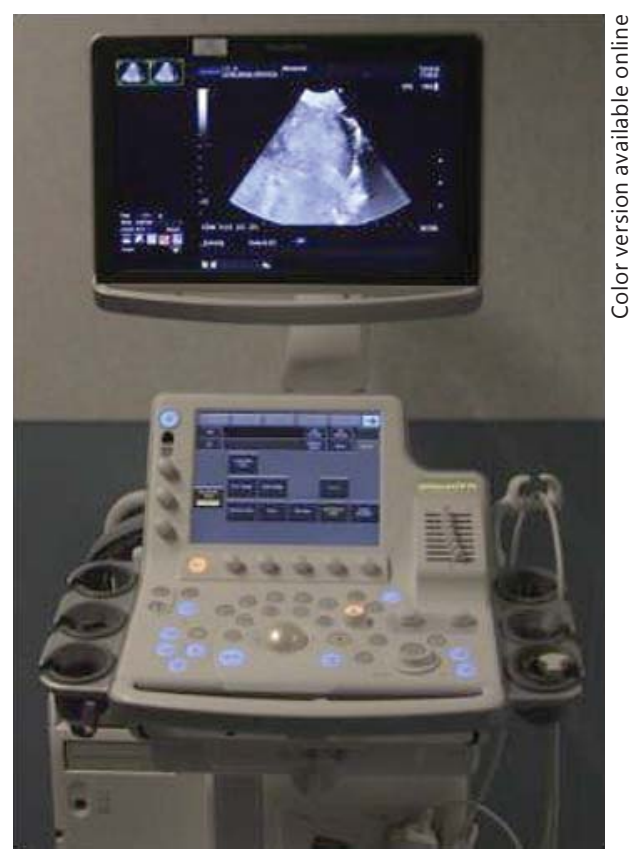

parenchyma and has been found to be the most accurate modality to characterize focal liver lesions $[10,11]$. Moreover, the hepatobiliary phase represents an added value in the detection and characterization of small nodules of uncertain nature [12]. The use of MRI over CT has also been endorsed by the American Hepato-Pancreato-Biliary Association in a recent consensus statement [13]. However, both CT and MRI have generally low sensitivity for small lesions $(<1 \mathrm{~cm})$, which are those that can modify the therapeutic strategy. For such small lesions, IOUS still remains the standard reference, and thus it should not be avoided in favor of any new preoperative imaging technique [14]. However, a certain decrease in the impact of IOUS in terms of staging is expected because such preoperative imaging techniques as CT and MRI improve rapidly. Notably, IOUS can detect up to $30 \%$ more nodules in cirrhotic livers; these are, however, more often regenerative nodules than tumors [15]. Indeed, IOUS in cirrhotic patients may overestimate the disease severity, and the performance of intraoperative biopsy cannot be the solution because of the low accuracy of frozen sections to differentiate early HCC from dysplastic or regenerative nodules. Figure 3a shows a HCC with the typical mosaic pattern, while figure $3 \mathrm{~b}$ shows a heterogeneous pattern of a cirrhotic liver with a hyperechoic lesion, which could easily be misjudged as pathological, but which has a low probability to really be an overt HCC [15]. To overcome this problem, besides the most accurate preoperative staging, the use of contrast-enhanced IOUS (CEIOUS) may be involved.

\section{CEIOUS}

Historically, carbon dioxide gas was initially used as contrast medium [16, 17]. Then, first-generation contrast media that enabled systemic circulation through pulmonary capillary vessels were developed, but with disparate results [18, 19]. Nowadays, secondgeneration contrast media, which are more sensitive and resistant to ultrasound, have been introduced [20,21]. The most used second-generation contrast medium is SonoVue (gaseous sulphur hexafluoride; Bracco, Milano Italy), which nowadays has a recognized diagnostic role for liver disease [22]. Recently, in Japan a new second-generation contrast medium has 
Donadon et al.: Intraoperative Ultrasound in Patients with Hepatocellular
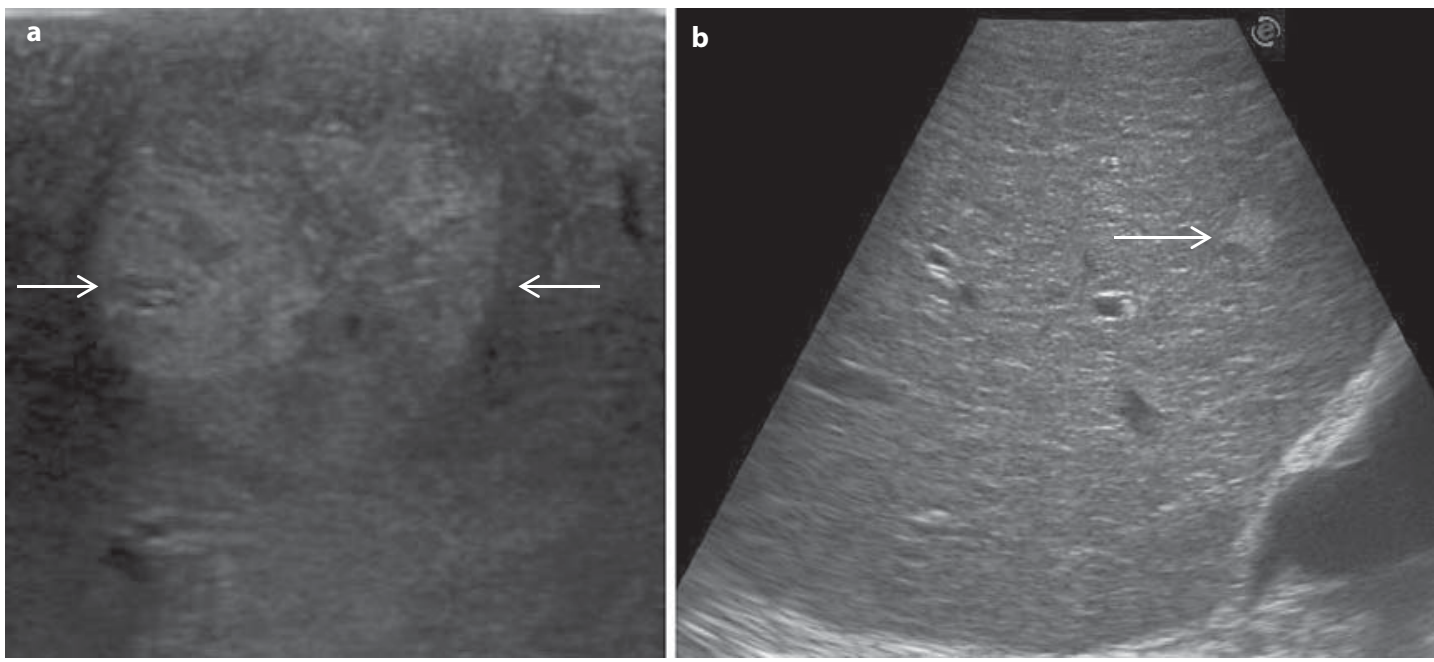

Fig. 3. a Typical mosaic pattern of HCC (arrows). b Liver with irregular echo pattern showing an hyperechoic nodule (arrow).

Fig. 4. Typical dual ultrasound image of HCC (arrows). a IOUS; b CEIOUS. fa = Feeding artery; P5-8 = portal pedicle to the right anterior section.
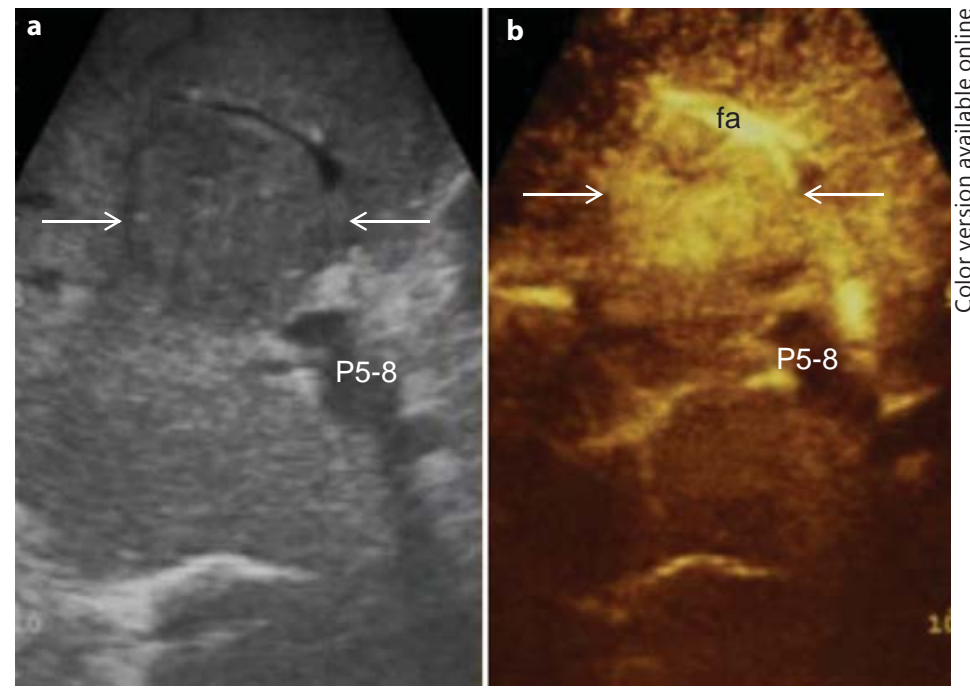

been used, Sonazoid (gaseous perflubutane; GE Healthcare, Norway), that has the advantage of Kupffer imaging in addition to vascular imaging, thus theoretically providing greater diagnostic accuracy [23].

Generally, in HCC CEIOUS is mainly used for the differential diagnosis of new lesions eventually detected at IOUS [24]. The rationale is, in fact, to improve the specificity of the basic IOUS in cirrhotic patients who present with multiple nodules. Such improvement in specificity may be reached studying the arterial phase of CEIOUS, namely malignant nodules with a typical arterial phase. Indeed, the neoangiogenesis of tumor nodules has been found to be one of the most specific criteria for differentiating HCC from regenerative or dysplastic nodules [25]. Of note, since the arterial phase lasts only 20-30 s, multiple injections of contrast medium may be required. Figure 4 shows a typical dual ultrasound imaging, i.e. basic IOUS and CEIOUS, of a HCC in its arterial phase. 


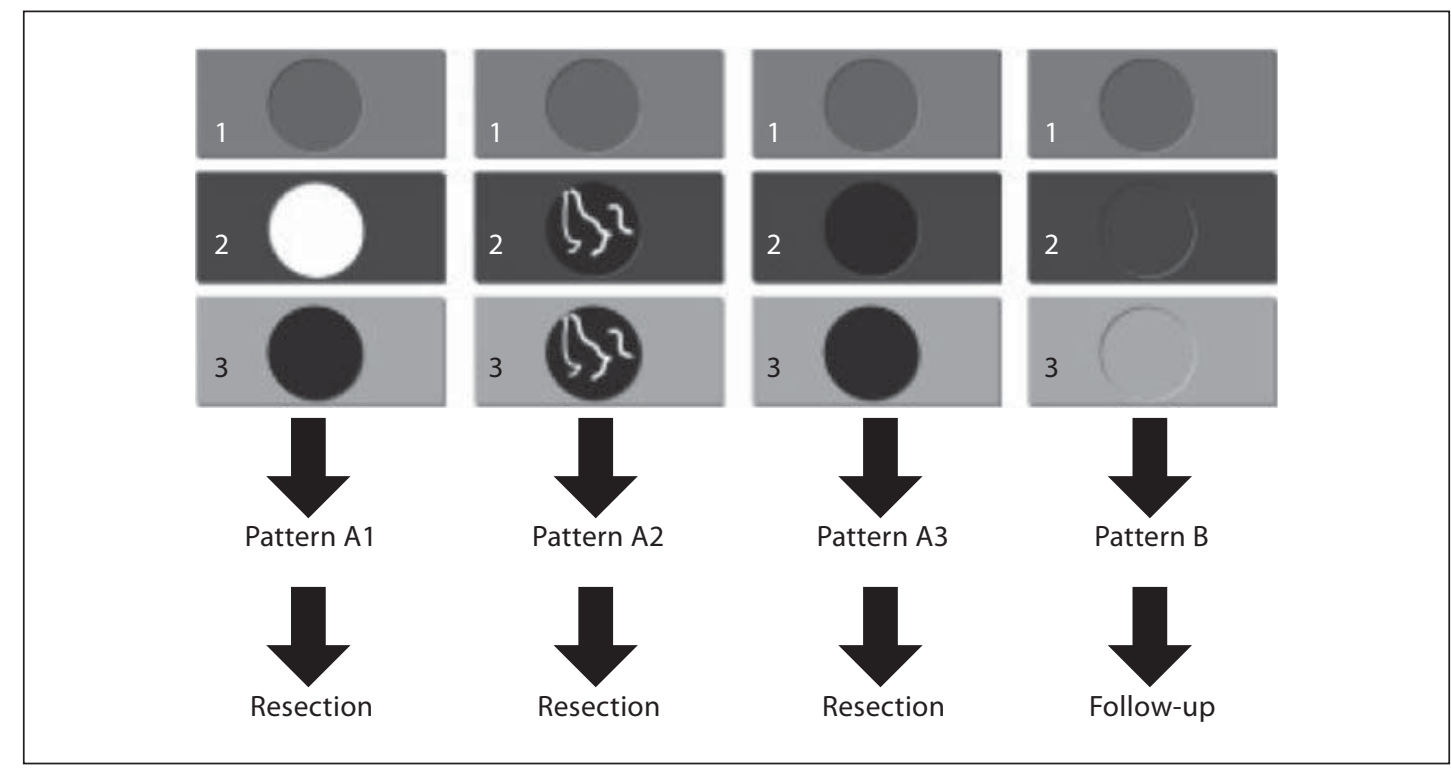

Fig. 5. Our classification of the various patterns of enhancement at CEIOUS in cirrhotic patients undergoing surgery for HCC. Four main patterns of CEIOUS can be recognized: A1: a typical wash-in and wash-out enhancement consistent with HCC. A2: a pattern with intranodular neoangiogenesis with wash-in and wash-out enhancement consistent with HCC. A3: a pattern of a hypoechoic nodule in any phase. B: a pattern of a nodule with the same enhancement of the surrounding liver parenchyma in any phase; this is not considered malignant.

In the percutaneous setting, the specificity of contrast-enhanced ultrasound in HCC has been reported by Quaia et al. [21] to be up to 95\%. In the intraoperative setting, CEIOUS is usually used to characterize small nodules, up to $1 \mathrm{~cm}$ in diameter, as generally larger nodules should have already been identified and characterized by preoperative imaging techniques. For such small nodules, the specificity is definitely lower than that reported in the percutaneous setting, and the criterion of vascularity may not be enough. For these reasons, Torzilli et al. [26] introduced a new classification to help the surgeon during the operative decision-making in patients with cirrhosis and multiple small nodules found at the basic IOUS (fig. 5). Using this classification, CEIOUS had a sensitivity of $100 \%$, a specificity of $69 \%$, and an overall accuracy of $80 \%$, and it modified the operative decision-making in up to $79 \%$ of the patients [26]; specificity is probably not that high, but is valid mostly for 1-cm lesions. New perspectives will definitely be provided by the use of Sonazoid as soon as it will be available worldwide.

\section{Guide for Resection}

Apart from disease staging, IOUS is essential to guide the resection strategy. It is almost impossible to correctly define the hepatic segmental boundaries without IOUS as well as the boundaries of the tumor itself also because of the existing wide variations in anatomy. In particular, in patients with cirrhosis the presence of irregular and macronodular surface as well as a relative hypertrophy of some parts of the liver makes the operation difficult. IOUS allows for three-dimensional reconstruction of the relationships between the tumor, the hepatic veins, and the Glissonian pedicles. Such pedicles are used as landmarks in the defi- 


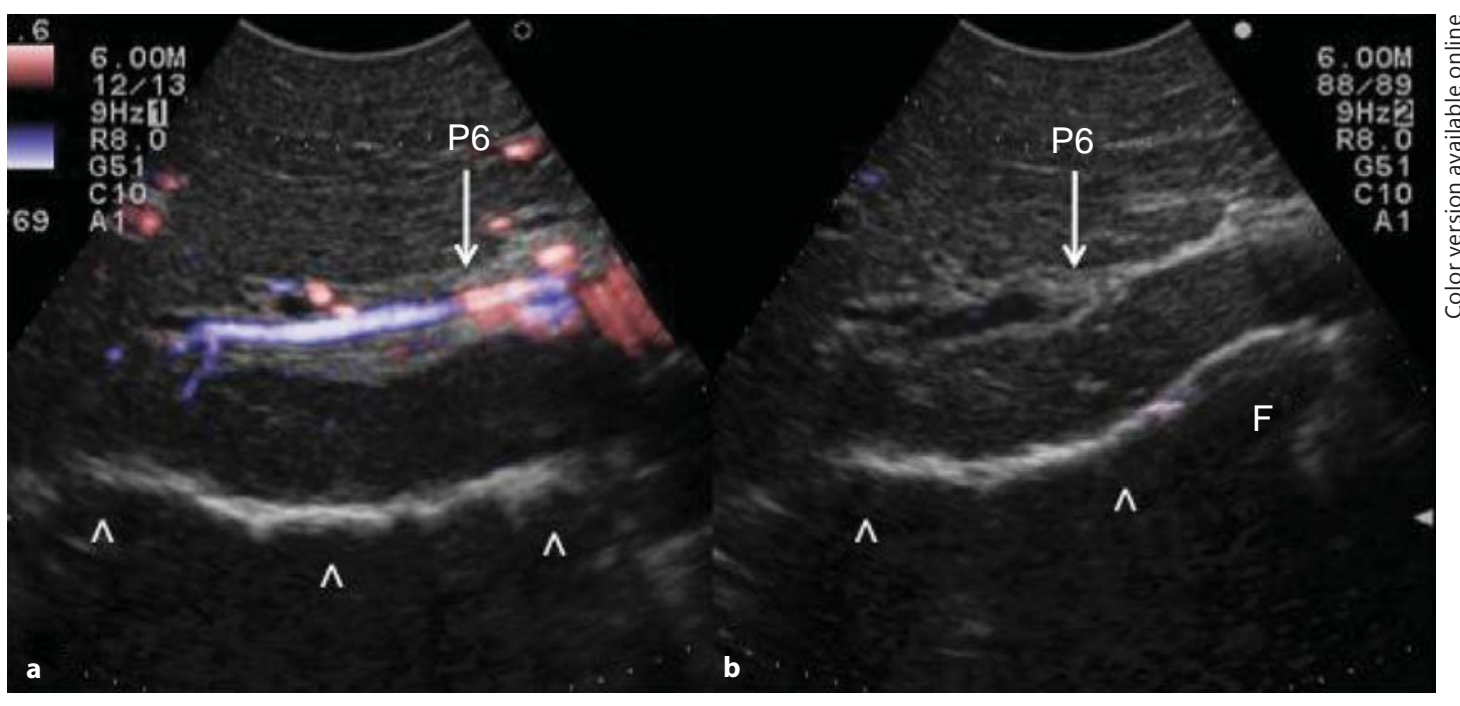

Fig. 6. Ultrasound-guided images of the finger compression maneuver for anatomical segmental resection. a The Glissonian pedicle for segment 6 is visualized (P6); b the Glissonian pedicle for segment 6 is compressed (P6). F = Finger. Arrowheads indicate the inferior part of the liver already mobilized.

nition of the resection line. Their localization is essential for planning the surgical strategy, which should be individualized in each patient. The main advantage of IOUS-guided resection is the modification of the traditional approach to liver tissue dissection, which involves dissection in vertical planes to avoid tumor exposure on the cut surface. With IOUS, the relationship between the dissection plane and the tumor edge can be followed in real time, and the direction of the dissection plane can be modified whenever needed. This approach has been recently redefined by Torzilli et al. [27] as the 'radical but conservative approach', and it should be applied in liver surgery to maximize the results. Also in patients who require major resections, IOUS allows for a better design of the dissection plane, leading to conservative surgery even in cases with complex presentations. Finally, specific and original IOUS techniques have already been developed to help the surgeon during the operation, as described below.

\section{Anatomical Resection by Ultrasound-Guided Finger Compression}

Initially used for tumors located in the left hemiliver [28], more recently ultrasoundguided finger compression has been successfully extended to any segment [29], including segment 8 and even to the sectional portion of the liver [30]. Once the feeding portal branch is identified at IOUS, it can be compressed using the IOUS probe by one side of the liver and the finger by the opposite side with the aim to induce a transient ischemia of the portion of the liver distally to the compression site. This portion can then be marked with the electrocautery, the compression can be released, and the resection carried out. This technique is simple, fast, noninvasive, and reversible. Moreover, the possibility to modify the site of compression and then the resection volume allows tailoring the resection based on tumor features and the status of the background liver. Yet, the compression can be used in a countercompression perspective that is the definition of the adjacent segmental margins to disclose those of the targeted segment. Indeed, for segments such as segments 1 and 4 superior for which the direct compression of the feeding portal branch could prove unfeasible, compressing the adjacent segmental branch allows for the definition of their segmental 

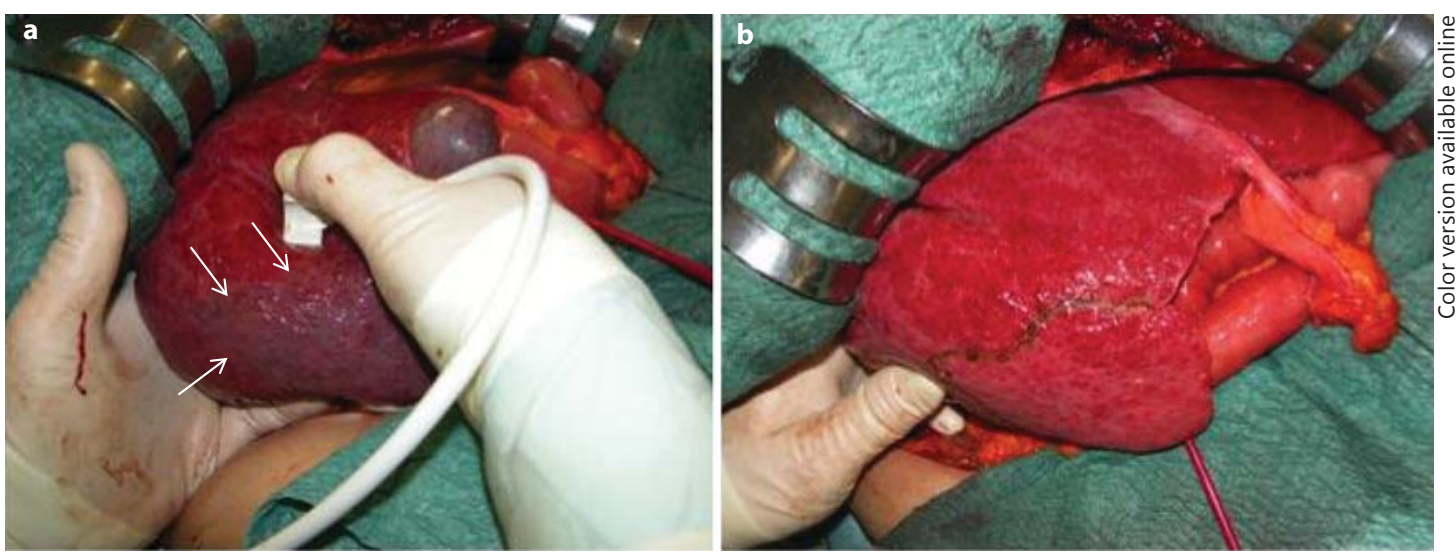

Fig. 7. Surgical images of the finger compression maneuver for anatomical segmental resection. a Anatomic ischemic demarcation of segment 6 (arrows) using the probe in the right hand on one side and a left hand fingertip on the opposite side. $\mathbf{b}$ The resection area which corresponds to the anatomical subsegmental portion as previously demarcated (arrows in a).

margins. Figure 6 shows the IOUS image of the ultrasound-guided finger compression of the portal branch for segment 6 , while figure 7 shows the corresponding ischemic demarcation in the surgical field.

\section{Conclusions and Future Trends}

This review has shown the importance of IOUS to stage liver diseases and to guide hepatectomy in patients with HCC. CEIOUS with SonoVue should be used to characterize any new nodule detected at IOUS, while CEIOUS with Sonazoid represents the next future area of studying because it promises a remarkable increase in sensitivity and specificity of the diagnostic tool.

The aforementioned surgical techniques allow for a precise and safe surgical resection of HCC, particularly for anatomical resection, as this is the mainstay for local treatment of HCC, and for liver parenchyma-sparing surgery to minimize postoperative complications. Thus, IOUS should be part of the mandatory ar mamentarium of the modern hepatic surgeon.

\section{Conflict of Interest}

None.

\section{References}

1 Adam R, Majno P, Castaing D, et al: Treatment of irresectable liver tumors by percutaneous cryosurgery. $\mathrm{Br}$ J Surg 1998;85:1493-1494.

2 Livraghi T, Giorgio A, Marin G, et al: Hepatocellular carcinoma and cirrhosis in 746 patients: long-term results of percutaneous ethanol injection. Radiology 1995;197:101-108.

3 Makuuchi M, Yamazaki S, Hasegawa H, et al: Ultrasonically guided liver surgery. Jpn J Ultrason Med 1980; 7:45-49.

4 Solbiati L, Livraghi T, Goldberg SN, et al: Percutaneous radiofrequency ablation of hepatic metastases from colorectal cancer in 117 patients. Radiology 2001;221:159-166. 
- 5 Makuuchi M, Torzilli G, Machi J: History of intraoperative ultrasonography. Ultrasound Med Biol 1998;24: 1229-1242.

6 Teh SH,Christein J, Donohue J, et al: Hepatic resection of hepatocellular carcinoma in patients with cirrhosis: Model of End-stage Liver Disease (MELD) score predicts perioperative mortality. J Gastrointest Surg 2005; 9:1207-1215.

- 7 Schroeder RA, Marroquin CE, Bute BP, et al: Predictive indices of morbidity and mortality after liver resection. Ann Surg 2006;243:373-379.

8 Donadon M, Torzilli G: Intraoperative ultrasound of the liver. AJR Am J Roentgenol 2012;198:W398.

- 9 Hata S, Imamura H, Aoki T, et al: Value of visual inspection, bimanual palpation, and intraoperative ultrasonography during hepatic resection for liver metastases of colorectal carcinoma. World J Surg 2011;35: 2779-2787.

10 Ayuso C, Rimola J, García-Criado A: Imaging of HCC. Abdom Imaging 2012;37:215-230.

11 Kim YK, Kwak HS, Kim CS, et al: Hepatocellular carcinoma in patients with chronic liver disease: comparison of SPIO-enhanced MR imaging and 16-detector row CT. Radiology 2006;238:531-541.

$\checkmark 12$ Sun HY, Lee JM, Shin CH, et al: Gadoxetic acid-enhanced magnetic resonance imaging for differentiating small hepatocellular carcinomas $(\leq 2 \mathrm{~cm}$ in diameter) from arterial enhancing pseudolesions. Special emphasis on hepatobiliary phase imaging. Invest Radiol 2010;45:96-103.

13 Vauthey JN, Dixon E, Abdalla EK, et al; American Hepato-Pancreato-Biliary Association; Society of Surgical Oncology; Society for Surgery of the Alimentary Tract: Pretreatment assessment of hepatocellular carcinoma: expert consensus statement. HPB (Oxford) 2010;12:289-299.

14 Hammerstingl R, Huppertz A, Breuer J, et al; European EOB-study group: Diagnostic efficacy of gadoxetic acid (Primovist)-enhanced MRI and spiral CT for a therapeutic strategy: comparison with intraoperative and histopathologic findings in focal liver lesions. Eur Radiol 2008;18:457-467.

15 Takigawa Y, Sugawara Y, Yamamoto J, et al: New lesions detected by intraoperative ultrasound during liver resection for hepatocellular carcinoma. Ultrasound Med Biol 2001;27:151-156.

16 Matsuda Y, Yabuuchi I: Hepatic tumors: US contrast enhancement with CO2 microbubbles. Radiology 1986; 161:701-705.

17 Kudo M, Tomita S, Tochio H, et al: Small hepatocellular carcinoma: diagnosis with US angiography with intraarterial CO2 microbubbles. Radiology 1992;182:155-160.

18 Blomley MJ, Albrecht T, Cosgrove DO, et al: Improved imaging of liver metastases with stimulated acoustic emission in the late phase of enhancement with the US contrast agent SH U 508A: early experience. Radiology 1999;210:409-416.

19 Heckemann RA, Cosgrove DO, Blomley MJ, et al: Liver lesions: intermittent second-harmonic gray-scale US can increase conspicuity with microbubble contrast material - early experience. Radiology 2000;216:592596.

20 Leen E, Angerson WJ, Yarmenitis S, et al: Multi-centre clinical study evaluating the efficacy of SonoVue (BR1), a new ultrasound contrast agent in Doppler investigation of focal hepatic lesions. Eur J Radio 2002; 41:200-206.

21 Quaia E, Calliada F, Bertolotto M, et al: Characterization of focal liver lesions with contrast-specific US modes and a sulfur hexafluoride-filled microbubble contrast agent: diagnostic performance and confidence. Radiology 2004;232:420-430.

-22 Piscaglia F, Lencioni R, Sagrini E, et al: Characterization of focal liver lesions with contrast-enhanced ultrasound. Ultrasound Med Biol 2010;36:531-550.

23 Arita J, Takahashi M, Hata S, et al: Usefulness of contrast-enhanced intraoperative ultrasound using sonazoid in patients with hepatocellular carcinoma. Ann Surg 2011;254:992-999.

24 Torzilli G: Contrast-enhanced ultrasonography during liver surgery. Br J Surg 2004; 91:1165-1167.

-25 Roncalli M, Roz E, Coggi G, et al: The vascular profile of regenerative and dysplastic nodules of the cirrhotic liver: implications for diagnosis and classification. Hepatology 1999;30:1174-1178.

-26 Torzilli G, Palmisano A, Del Fabbro D, et al: Contrast-enhanced intraoperative ultrasonography during surgery for hepatocellular carcinoma in liver cirrhosis: is it useful or useless? A prospective cohort study of our experience. Ann Surg Oncol 2007;14:1347-1355.

27 Torzilli G, Montorsi M, Donadon M, et al: 'Radical but conservative' is the main goal for ultrasonographyguided liver resection: prospective validation of this approach. J Am Coll Surg 2005;201:517-528.

28 Torzilli G, Makuuchi M: Ultrasound-guided finger compression in liver subsegmentectomy for hepatocellular carcinoma. Surg Endosc 2004;18:136-139.

29 Torzilli G, Procopio F, Cimino M, et al: Anatomical segmental and subsegmental resection of the liver for hepatocellular carcinoma: a new approach by means of ultrasound-guided vessel compression. Ann Surg 2010;251:229-235.

-30 Torzilli G, Procopio F, Palmisano A, et al: New technique for defining the right anterior section intraoperatively using ultrasound-guided finger counter-compression. J Am Coll Surg 2009;209:e8-e11. 